\title{
PSEUDO-ARGYLL ROBERTSON PUPILS IN OPTIC NERVE DISEASE*
}

\author{
BY \\ PAUL LEVATIN AND ROBERT J. OGILVIE \\ Departments of Ophthalmology and Pathology, Permanente Medical Group, Kaiser Foundation Hospital, \\ Oakland, California, U.S.A.
}

THE essentials of the Argyll Robertson pupil are absolute immobility to light and unimpaired response to the accommodation-convergence, or near, reflex. When, in addition, the pupils are less than $3 \mathrm{~mm}$. in diameter, unequal, and irregular they are, according to Duke-Elder (1949), pathognomonic of tabes or general paresis. Can the tabetic pupil be diagnosed if the classic signs are modified or absent?

Apparently it is not necessary for the pupil to be immobile to light, for in the incomplete Argyll Robertson (AR) syndrome due to syphilis the light reflex is reduced but not absent. However, recent information suggests that unless the pupils are miotic and unequal it is unsafe to make a diagnosis of the AR syndrome. In his outstanding studies in pupillography, Lowenstein (1954) has shown that partial lesions of the retina or optic nerve are associated with a normal pupil reaction to near vision but a reduced or "low intensity" light reflex, characterized by an inextensive contraction with premature redilation. These reactions superficially resemble those seen in neurosyphilis but differ in that "the light reflex in the AR pupil, if present at all, is extremely sluggish and usually lasts longer than normal" (Lowenstein, 1956a). Moreover, the pupils in optic nerve disease are round, equal, and larger than normal. Thus optic nerve lesions produce pseudo-AR pupils, differing from AR pupils in both appearance and reaction to light.

In a previous paper (Levatin, 1959) and motion picture (Levatin, 1962), it was shown that the pupillographic "low intensity" reaction is visible as the Marcus Gunn sign of pupillary escape - the pupil of the affected eye redilating or escaping from the light more rapidly than does that of the normal eye in response to the same illumination. Elaborate apparatus is not required to distinguish between the decreased light reflex in the AR pupil and the pupillary escape in optic nerve disease. Clinically, the Marcus Gunn sign may be readily demonstrated by the swinging flashlight test:

The patient is asked to look steadily at a distant point to eliminate the effect of accommodation on pupil size. The room is darkened and a bright light is passed back and forth from one eye to the other. The physician observes the movement of the illuminated pupil, which becomes larger in the abnormal eye and smaller in the normal eye.

This apparently contradictory reaction - a pupil dilating when exposed to illumination, instead of contracting-is the more striking, the greater the difference of light sense in the two eyes (Levatin, 1961). Since it depends on the decrease in light sense produced by all lesions of the retina or optic nerve, pupillary escape is a valuable diagnostic sign (Drews, 1962).

The following case of a slow-growing brain lesion undiagnosed during life illustrates an incorrect diagnosis of AR pupils. In optic nerve disease the pupils may be thought to be immobile when in reality they show pupillary escape.

* Received for publication April 25, 1963. 


\section{Case Report}

A 49-year-old labourer came to the out-patients department in January, 1949, complaining that for 7 years he had suffered from episodes in which the left side of his face would "draw up" with twitching and closing of the left eyelids and engorgement of the neck veins. Attacks of unconsciousness, lasting about 5 minutes, during which he "twitched and trembled" had been present since 1945. Defective vision had been present in his left eye since 1943. There was no history of syphilis or antisyphilitic treatment.

Neurological examination revealed right mimetic facial paresis and an occasional spasm of the muscles of the left side of the face lasting a few seconds. The pupils reacted in accommodation but were fixed to light. The optic discs were white and their nasal margins were noted by one observer to be somewhat rough. The patient swayed slightly in the Romberg test but did not fall. When the eyes were closed the outstretched arms tended to drift outward.

The blood Kline and Kahn tests were negative. The cerebrospinal fluid (opening pressure $270 \mathrm{~mm}$.) showed 1 white blood cell, a negative Kolmer test, a colloidal gold curve of 0001110000 , and a total protein of $65 \mathrm{mg}$. per $100 \mathrm{ml}$. In the plain roentgenograms of the skull the sella turcica measured $15 \times 17 \mathrm{~mm}$. with slight erosion of the posterior clinoids and a questionable calcification at the level of the dorsum sellae.

Several specialists concluded that the patient had Argyll Robertson pupils and optic atrophy due to tabes dorsalis, but also $x$-ray changes suggestive of a lesion in the area above the sella.

While the patient was in the hospital awaiting air studies, an ophthalmologic consultation was obtained. The visual acuity of the right eye was 20/20; that of the left was $2 / 200$ and could not be improved. The pupils were round and $4 \mathrm{~mm}$. in diameter. They reacted to light promptly through a narrow arc, redilating quickly, the left pupil through a narrower arc than the right*. The reaction on accommodation was normal. The optic discs were pale (the left much more than the right) and were elevated one dioptre. A grey veil of glial tissue overlay the disc, rendering the lamina cribrosa invisible and making the disc edges hazy and irregular. There was slight narrowing of the arteries in the right fundus; marked narrowing of the arteries in the left fundus resulted in an artery to vein ratio of 1:4. Some of the vessels were sheathed near the disc edges. Perimetry revealed generalized contraction of the peripheral fields and enlargement of the blind spots (Fig. 1). The diagnosis of bilateral secondary optic atrophy was made.

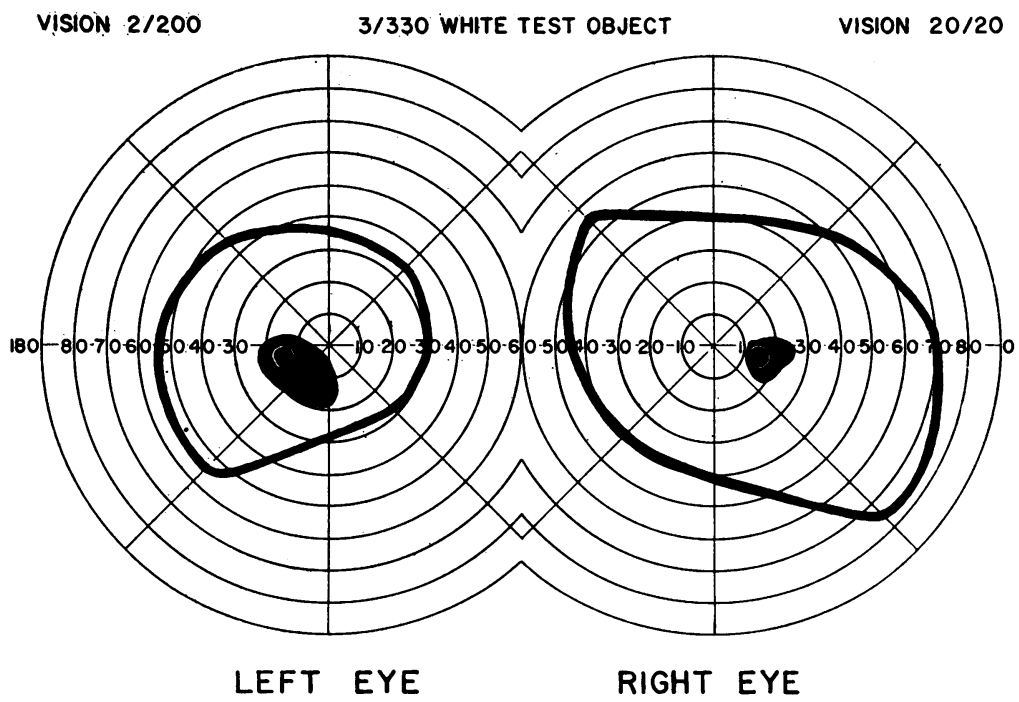

FIG. 1.-The right peripheral field shows a generalized contraction to seven-eighths normal size and the blind spot is enlarged four times; the left peripheral field is contracted to one-half normal size and the blind spot is enlarged ten times. 
Pneumo-encephalography was not considered diagnostic, as insufficient air entered the ventricular system completely to outline it (Fig. 2). A repeat procedure a week later again showed only partial filling of the lateral ventricles. Ventriculography (Fig. 3) revealed a huge but symmetrical dilation of all the ventricles but no evidence of a tumour mass.

Fig. 2.-Pneumo-encephalogram: Air only partially filled the lateral ventricles.
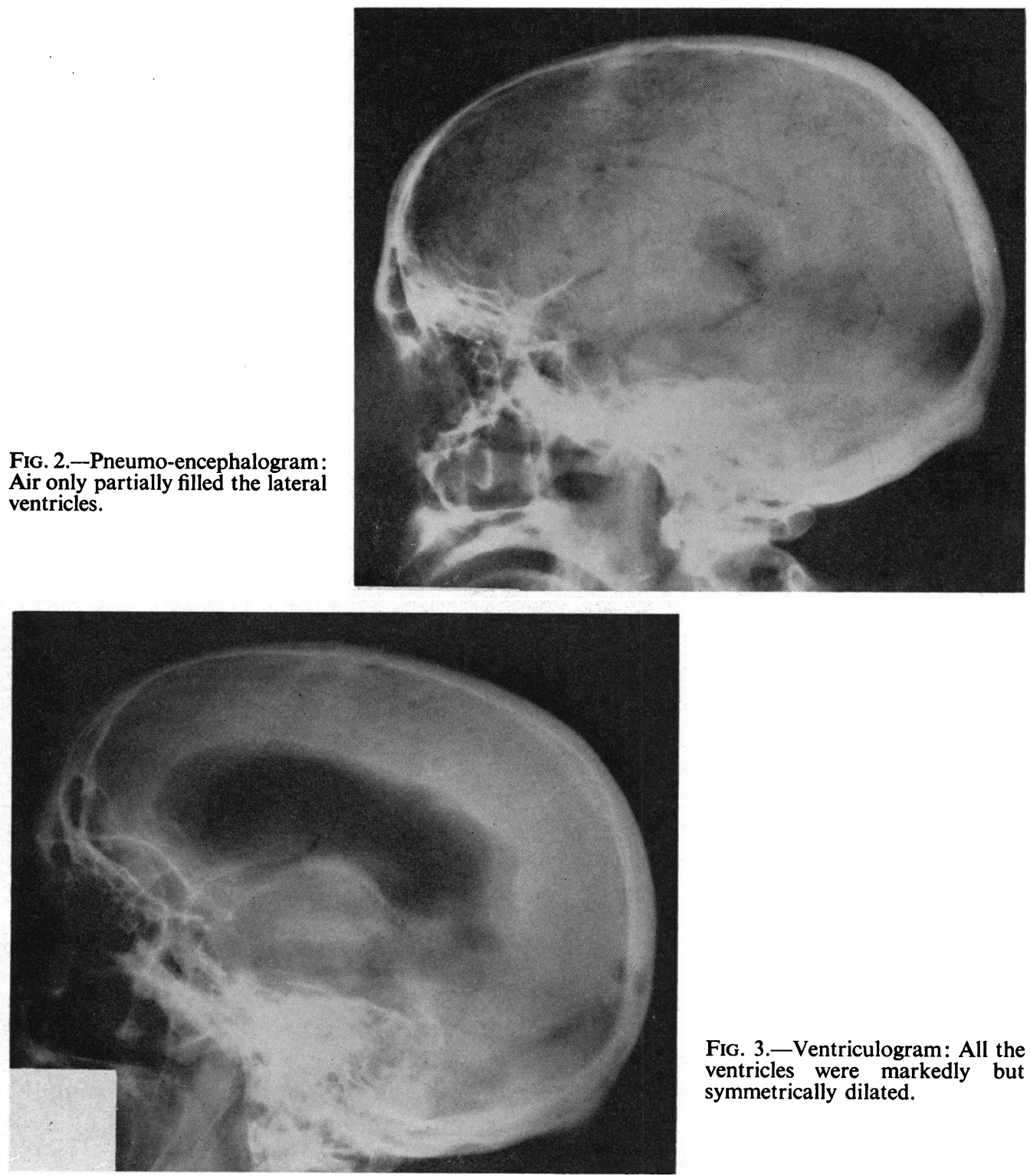

FIG. 3.-Ventriculogram: All the ventricles were markedly but symmetrically dilated.

It was felt that several years previously mild chronic inflammation had gradually blocked the outflow of cerebrospinal fluid from the fourth ventricle, resulting in increased intracranial pressure, chronic papilloedema with secondary optic atrophy, internal hydrocephalus, enlargement of the sella turcica, and epileptiform seizures. Since the pneumo-encephalograms had been partially successful, it was concluded that the block was no longer present.

Dilantin was prescribed; the patient returned to work and for 2 years was free of seizures. By April, 1955, the visual acuity in the right eye had decreased to 20/50-2; that of the left eye remained 
$2 / 200$. The optic discs had a slight, unmeasurable elevation and were pale, with two capillaries on the right disc and none on the left. Repeat skull $x$ rays, when compared with those taken in 1949 , showed increased demineralization or destruction of the posterior clinoids. Repeat ventriculography was recommended but was refused.

Both grand mal and petit mal seizures became frequent. Ataxia increased until the patient could walk only with a cane. The last ophthalmological examination, in January, 1957, revealed little change in the visual acuity or in the other eye findings. The patient died suddenly in September, 1958 , after an episode of unconsciousness.

Post mortem Examination.-The positive findings were confined to the brain. Arising from the roof of the fourth ventricle, displacing the vermis of the cerebellum cephalad and the hemispheres of the cerebellum laterally, was an irregular, firm, somewhat gritty mass measuring $4 \times 3 \times 2.5 \mathrm{~cm}$. (Fig. 4). It could be readily shelled out from the contiguous cerebellar tissue. All the ventricles were considerably distended.

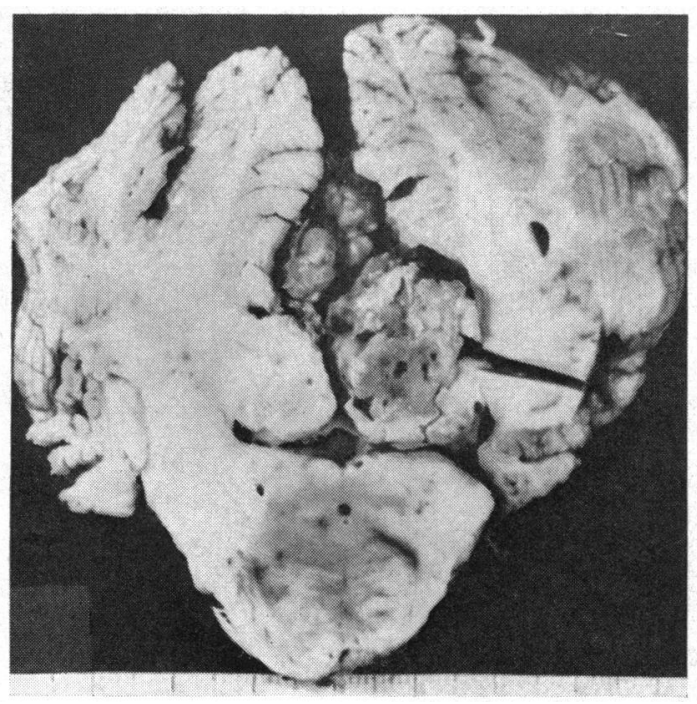

Fig. 4.-A large tumour arising from the roof of the fourth ventricle forced apart the hemispheres of the cerebellum (scale in inches).

Microscopic examination revealed that the tumour contained an abundance of glial fibrils tending occasionally to show some whorled appearance (Fig. 5, opposite). The characteristics of this glioma were considered compatible with either an ependymoma or an astrocytoma, in some areas distinctly favouring the former diagnosis.

\section{Comment}

That the syphilitic lesion which produces the AR pupil does not impair optic nerve conductivity or vision is indicated by the first phrase in Argyll Robertson's description (1869), "Although the retina is quite sensitive, and the pupil contracts during the act of accommodation for near objects, yet an alteration of the amount of light admitted into the eye does not influence the size of the pupil" (italics ours). In the four cases upon which Argyll Robertson based his definition, the patients had small pupils (spinal miosis) and good vision; whereas syphilitic patients with primary (tabetic) optic atrophy have large pseudo-AR pupils and poor vision. The coexistence of AR pupils with tabetic optic atrophy assumed in our case must be rare, as it requires separate lesions in the optic nerves and in the midbrain at the presumed site (Lowenstein, 1956b) of the lesion causing the AR pupil. 
FIG. 5.-One of the more cellular portions of the tumour, illustrating perivascular distribution of cells and a slight tendency towards curved fasciculi. Haematoxylin and eosin. $\times 60$.

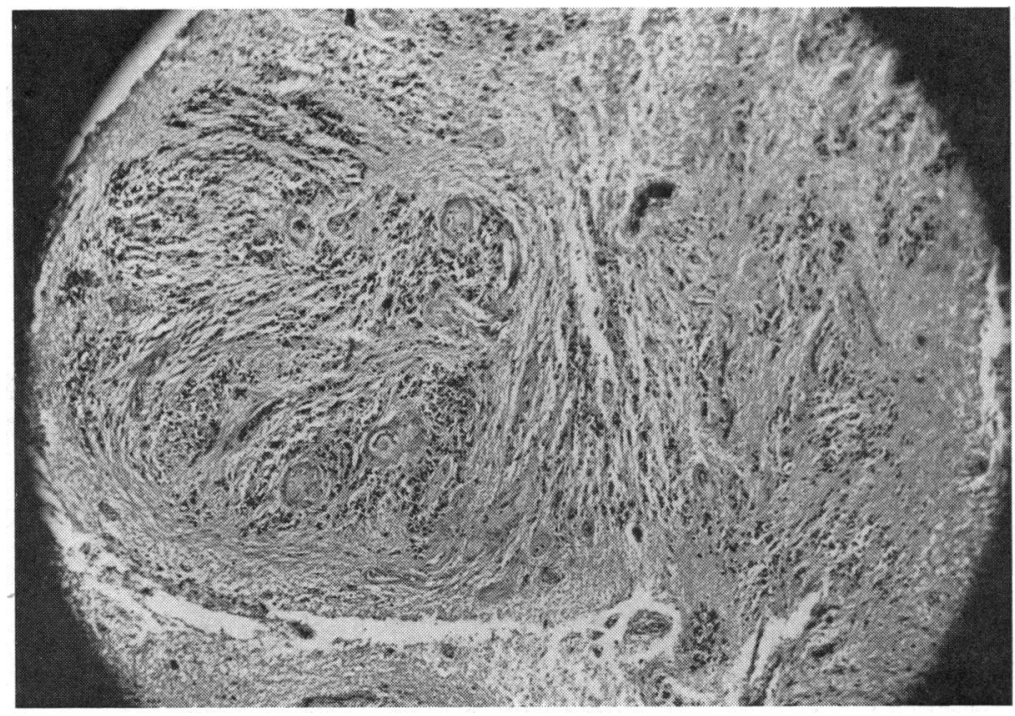

Summary

Optic nerve disease produces pseudo-Argyll Robertson pupils that differ from those described by Argyll Robertson in that they are large, round, equal, and show the Marcus Gunn sign of pupillary escape-an inextensive, unsustained contraction of the pupils in bright illumination. Pupillary escape is an index of optic nerve conductivity and thus a diagnostic sign of optic nerve impairment. It may be readily elicited in a rapid and simple manner by the swinging flashlight test.

The clinical importance of these concepts is illustrated by an ependymoma of the fourth ventricle, present at least 16 years, which had produced a partial block in the ventricular system and a compensated internal hydrocephalus. Tabes dorsalis was diagnosed because optic atrophy secondary to ancient papilloedema was considered to be primary (tabetic) optic atrophy; and large, equal, pseudo-AR pupils showing pupillary escape were mistaken for immobile AR pupils.

The coexistence of AR pupils and tabetic optic atrophy is unlikely, because it depends upon multiple lesions in the central nervous system.

\section{Conclusion}

One can avoid confusing the incomplete AR syndrome of syphilis with the pseudoAR pupils of optic nerve disease by making the diagnosis of the AR syndrome only when the pupils are miotic and unequal, and do not show pupillary escape.

We wish to express our gratitude to Dr. Melvin Friedman for the photomicrograph and for his help in its interpretation.

\section{REFERENCES}

Drews, R. C. (1962). Amer. J. Ophthal., 54, 1109.

DUKe-Elder, S. (1949). "Text-Book of Ophthalmology", vol. 4, p. 3784 . Kimpton, London.

LeVatin, P. (1959). A.M.A. Arch. Ophthal., 62, 768. (1961). Amer. J. Ophthal., 51,1308.

(1962). Motion Picture, "Swinging Flashlight Test for Retinal or Optic Nerve Disease", $16 \mathrm{~mm}$. sound film. University of California Extension Division, Berkeley.

Lowenstein, O. (1954). A.M.A. Arch. Ophthal., 52, 385.

(1956a). Ibid., 55, 356.

- (1956b). Amer. J. Ophthal., 42, no. 4, Part II, p. 105.

Robertson, D. ARgYLl (1869). Edinb. med. J., 15, 487. 